\title{
EUCLIDEAN GAUGE FIELDS IN FOUR DIMENSIONS FROM STOCHASTIC DIFFERENTIAL EQUATIONS
}

\author{
R. VILELA MENDES \\ CFMC, Instituto Nacional de Investigação Cientifica, Av. Gama Pinto 2, P-1699 Lisboa Codex, Portugal
}

Received 5 November 1987

\begin{abstract}
Using a stochastic differential equation for field configurations in a three-dimensional space lattice, one proves existence of an euclidean theory for non-abelian gauge fields on the lattice. The theory is also shown to possess a scaling limit for the mass gap at weak coupling. The construction is extended in a less rigorous manner to a theory with fermions and gauge fields.
\end{abstract}

The formal analogy between the time evolution of the probability density in the Schrödinger picture and the Fokker-Planck equation was used in refs. [1-3] to construct stochastic processes in configuration space associated to lattice gauge theories. From the corresponding stochastic differential equations (SDE) mass gaps and euclidean correlations may be measured.

An important point was the proof of existence of stochastic processes which, in the classical continuum limit, lead to the same hamiltonian (as a generator of the process semigroup) but nevertheless correspond to nonequivalent classes of dynamics [3]. Non-equivalence is proven by showing that at weak coupling the mass gaps follow different scaling laws.

These results strongly suggest that, for systems with infinite number of degrees of freedom, hamiltonian or lagrangian functions provide only an incomplete specification of the theory. A specification through SDEs might be more satisfactory in the sense that an SDE is closer to provide a control over probability measures and asymptotic behaviour of operators than the formal measure that one defines by the exponential of the action.

In this paper we give first steps on the way to a rigorous construction of four-dimensional non-trivial euclidean fields on the lattice and their continuum limits, using SDEs in configuration space. First to be discussed is an equation for non-abelian fields on the lattice. Besides supplying a proof of consistency of the lattice model, one also sees that it satisfies a necessary condition for the existence of an asymptotically free continuum limit. Then we discuss the inclusion of fermion fields, as a concrete example of the fermion scheme developed in ref. [2]. This involves a stochastic equation with both diffusion and jumping.

Consider a space lattice $\mathbb{Z}_{a}^{3}=\left\{a n \mid n \in \mathbb{Z}^{3}\right\}$ and let $\Lambda_{a}=\Lambda \cap \mathbb{Z}_{a}^{3}$ where $A$ is a bounded subset of $\mathbb{R}^{3}$.

A gauge field configuration is a mapping from the links to the space of a compact Lie group:

$l \rightarrow U_{l}=\operatorname{exp~i~}\left(\theta^{\alpha}, \xi^{\alpha}\right)$,

the $\xi^{\alpha}$ 's being a Lie algebra basis and $\theta^{\alpha}{ }_{i} \in \mathrm{S}^{1}$.

To specify that a link $l$ connects the sites $n$ and $n+i$ along the unit vector $i$ one uses the notation $\theta^{\alpha}{ }_{l} \equiv \theta_{n, n+i}^{\alpha}$.

In $A_{a}$ one considers the following SDE:

$\mathrm{d} \theta^{\alpha}{ }_{l}=\mathrm{i} b^{\alpha}, \mathrm{d} s+(g / \sqrt{a}) \mathrm{d} W^{\alpha}{ }_{l}$

with $\left\langle\mathrm{d} W^{\alpha}{ }_{l} \mathrm{~d} W^{\beta}{ }_{l}\right\rangle=\delta^{\alpha \beta} \delta_{l l} \mathrm{~d} s$ and 


$$
\begin{aligned}
& \mathrm{i} b_{n, n+i}^{\alpha}=(1 / 2 a) F^{\prime}\left(\sigma_{\mathrm{G}}\right)\left(Q^{\alpha}{ }_{n, n+i}+Q^{\alpha}{ }_{n+i, n+2 i}\right) h^{\prime}\left(\theta^{\alpha}{ }_{n, n+i}\right), \\
& Q_{n, n+i}^{\alpha}=-\frac{1}{4} \gamma_{i j k}\left[h\left(\theta_{n+j, n+j+k}^{\alpha}\right)+\frac{1}{2} f^{\alpha \beta \gamma} h\left(\theta_{n, n+j}^{\beta}\right) h\left(\theta^{\prime}{ }_{n, n+k}\right)\right], \\
& \gamma_{i j k}=(\operatorname{sign} i)(\operatorname{sign} j)(\operatorname{sign} k) \varepsilon_{|i| j|k|}, \\
& \sigma_{\mathrm{G}}=\frac{1}{8} g^{2} \operatorname{Tr}\left(\sum_{n} \sum_{i j k} \gamma_{i j k}\left[-\boldsymbol{h}\left(\theta_{n, n+i}\right) \boldsymbol{h}\left(\theta_{n+j, n+j+k}\right)+\mathrm{i} \frac{2}{3} \boldsymbol{h}\left(\theta_{n, n+i}\right) \boldsymbol{h}\left(\theta_{n, n+j}\right) \boldsymbol{h}\left(\theta_{n, n+k}\right)\right]\right) .
\end{aligned}
$$

$h(\theta)$ is a continuous function in $\mathrm{S}^{1}$ such that $h^{\prime}(0)=1$ and $h^{\prime \prime}(\theta) \rightarrow 0$ faster than $\theta^{2}$ when $\theta \rightarrow 0$. For example $h(\theta)$ may be a $2 \pi$-periodic piecewise linear function of slope $\pm 1, F\left(\sigma_{\mathrm{G}}\right)$ is a continuous piecewise linear function of $\sigma_{\mathrm{G}}$ such that $F^{\prime}\left(\sigma_{\mathrm{G}}\right)= \pm 1$ a.e. and $F\left(\sigma_{\mathrm{G}}\right)$ is bounded from above and below; $F_{\min } \leqslant F\left(\sigma_{\mathrm{G}}\right) \leqslant F_{\max } \cdot \boldsymbol{h}(\theta)$ means $h\left(\theta^{\alpha}\right) \xi^{\alpha}$.

Considering the formal continuum limit $(a \rightarrow 0)$ with

$\boldsymbol{A}^{\mu}(n)=(1 / g a) \boldsymbol{S}^{\mu}(n)=\left(\boldsymbol{\theta}_{n-\mu, n}+\boldsymbol{\theta}_{n, n+\mu}\right) / 2 g a$,

$\partial_{j} \boldsymbol{A}^{k}(n)=\left(1 / 2 g a^{2}\right)\left[\boldsymbol{S}^{k}(n+j)-\boldsymbol{S}^{k}(n-j)\right]$,

one recognizes in the RHS of eq. (4) the lattice version of the Chern-Simons density and the drift $\mathrm{i} b^{\alpha}$, becomes the chromomagnetic field parallel to the link $l$. The regularizing functions $h(\theta)$ and $F\left(\sigma_{G}\right)$ (equal to one in the $\theta \rightarrow 0$ limit) are required to ensure global existence of the solution to eq. (1) and a consistent quantum continuum limit (see below) ${ }^{\# 1}$. The drift is related to $F\left(\sigma_{\mathrm{G}}\right)$ as follows:

$\mathrm{i} b^{\alpha},=\left(g^{2} / a\right)\left(\partial / \partial \theta^{\alpha}\right) F\left(\sigma_{\mathrm{G}}\right)$.

Eq. (1) is a SDE with values in $\otimes_{n i} S^{i}, n_{l}$ being the number of links in $A_{a}$. Existence and uniqueness of a solution may be obtained either by patching up the solutions in coordinate neighbourhoods or using Whitney's imbedding theorem and considering the corresponding process in $\mathbb{R}^{2 n_{i}+1}$.

In eq. (1) the diffusion coefficient is a constant and the drift a bounded continuous function. Therefore by theorem 2.2 in chapter IV of ref. [4] there is a solution for any given initial probability distribution of $\theta(0)$. Furthermore the drift being locally Lipschitz continuous there is pathwise uniqueness and a unique strong solution of eq. (1) (theorem 3.1 in chapter IV of ref. [4]).

Next one discusses the invariant measure of the process. Because the drift is a gradient [see eq. (6)] the function

$\rho(\theta)=c \exp \left[\left(2 g^{2} / a\right) F\left(\sigma_{\mathrm{G}}\right)\right]$

is an invariant density. $c$ is the normalization constant, $c=\int \exp \left[\left(2 g^{2} / a\right) F\left(\sigma_{\mathrm{G}}\right)\right] \mathrm{d} \mu(\boldsymbol{\theta})$, the integration being over $\otimes_{n} S^{\prime}$.

To prove uniqueness and ergodicity of the invariant measure one has to discuss the recurrence of the stochastic process. By Whitney's theorem one imbeds $\bigotimes_{n i} S^{1}$ into $\mathbb{R}^{2 n+1}$ as a closed bounded submanifold $M$. Let $\Gamma$ be a bounded set in $\mathbb{R}^{2 n+1}$ containing $\mathbf{M}$ and larger than $\mathrm{M}$ in the sense $\partial \Gamma \cap \partial \mathbf{M}=\emptyset$. Let $\mathbf{U}_{1}$ be an arbitrary set in $\mathrm{M}$ and $\mathrm{U}=\Gamma \backslash \mathrm{U}_{1}$. Then $\mathrm{U}$ is a set bounded in all coordinates, i.e. $x \in \mathrm{U} \Rightarrow x_{i}^{\mathrm{min}} \leqslant x_{i} \leqslant x_{i}^{\max }$. By the corollary 2 of theorem 7.1, chapter III of ref. [5], for any $x \in \mathrm{U} \cap \mathrm{M}$,

$P_{x}\left\{\tau_{\mathrm{U}}<\infty\right\}=1$,

and furthermore $\tau_{U}$ (the first hitting time of the boundary $\partial U$ ) has moments of all orders. Because the process is restricted to $M$, for an initial point in $U \cap M$, the boundary point of $U$ that is hit is also a boundary point of $\mathrm{U}_{1}$. Therefore the Markov process in M associated to the SDE (1) is positively recurrent. Hence, by theorem

${ }^{\# 1}$ Defining the variables $\theta^{\alpha}$, in $R$ instead of $S^{1}$ and choosing for $h(\theta)$ the identity function [ 3 ] one could still assert the existence of local solutions, but not global solutions without explosions. 
5.1 (and corollaries) in chapter IV of ref. [5] one concludes that the invariant measure with density (7) is unique and the law of large numbers holds in the sense

$P\left(\frac{1}{T} \int_{0}^{T} f(\theta(t)) \mathrm{d} t \underset{T \rightarrow \infty}{\longrightarrow} \int f(\theta) \rho(\theta) \mathrm{d}^{n} \theta\right)$.

The measure $\mathrm{d} \rho=\rho(\theta) \prod_{l} \mathrm{~d} \theta_{l}$, being invariant and ergodic, it provides a suitable vacuum measure. Consider the space of square integrable functions for the measure $\mathrm{d} \rho$, i.e. $\mathscr{H}_{\mathrm{D}}=\mathrm{L}_{2}(F(\theta), \mathrm{d} \rho) . \mathscr{H}_{\mathrm{D}}$ is the Dirichlet representation $\$ 2$ of the euclidean theory.

Existence and uniqueness of the solution to the SDE (1) ensures the existence of a Markov process with values in $\otimes_{n} S^{\mathrm{l}}$ and a well-defined set of transition functions $P\left(t, \theta_{1}, \mathrm{~d} \theta_{2}\right)$ (where $\mathrm{d} \theta_{2}$ denotes the flat measure in $\otimes$ ${ }_{n} S^{1}$ ). These are used to characterize the euclidean time correlations of the theory (Schwinger functions) by

$$
\begin{aligned}
& \left\langle F_{n}\left(\theta\left(t_{n}\right)\right) F_{n-1}\left(\theta\left(t_{n-1}\right)\right) \ldots F_{0}\left(\theta_{0}\right)\right\rangle \\
& \quad=\int F_{N}\left(\theta_{n}\right) P\left(t_{n}-t_{n-1}, \theta_{n-1}, \mathrm{~d} \theta_{n}\right) F\left(\theta_{n-1}\right) P\left(t_{n-1}-t_{n-2}, \theta_{n-2}, \mathrm{~d} \theta_{n-1}\right) \ldots F_{0}\left(\theta_{0}\right) \mathrm{d} \rho\left(\theta_{0}\right),
\end{aligned}
$$

where $t_{n} \geqslant t_{n-1} \geqslant \ldots \geqslant 0$ and the $F$ 's are bounded functions.

The transition functions are probability measures on the last argument, therefore the action of the process semigroup $T(t)$ at each step, for example

$\left(T\left(t_{n}-t_{n-1}\right) F_{n}\right)\left(\theta_{n-1}\right)=\int F_{n}\left(\theta_{n}\right) P\left(t_{n}-t_{n-1}, \theta_{n-1}, \mathrm{~d} \theta_{n}\right)$,

preserves the boundedness of the integrand functions and ensures the existence of the Schwinger functions, provided the $F$ 's are bounded functions in $\otimes_{n t} S^{1}$. Furthermore ergodicity of the process [eq. (8)] allows the determination of the Schwinger functions from time averages.

The generator of the Markov process is

$H_{\mathrm{D}}=-\frac{g^{2}}{2 a} \sum_{l, \alpha} \frac{\partial}{\partial \theta^{\alpha}} \frac{\partial}{\partial \theta^{\alpha}},-\sum_{l, \alpha} \mathrm{i} b^{\alpha}, \frac{\partial}{\partial \theta^{\alpha}}$.

The unitary transformation $H_{\mathrm{D}} \rightarrow H_{\mathrm{G}}=\rho^{1 / 2} H_{\mathrm{D}} \rho^{-1 / 2}$ between the spaces $\mathscr{H}_{\mathrm{D}}=\mathrm{L}_{2}(F(\theta), \mathrm{d} \rho)$ and $\mathscr{H}_{\mathrm{S}}=\mathrm{L}_{2}\left(\rho^{1 / 2} F(\theta)\right.$, $\mathrm{d} \theta$ ) leads to the hamiltonian operator

$H_{\mathrm{G}}=\frac{g^{2}}{2 a} \sum_{l, \alpha}\left(-\frac{\partial}{\partial \theta^{\alpha}}-\mathrm{i} \frac{a}{g^{2}} b^{\alpha}{ }_{l}\right)\left(\frac{\partial}{\partial \theta^{\alpha}}-\mathrm{i} \frac{a}{g^{2}} b^{\alpha}{ }_{l}\right)$.

Using the replacement $\partial / \partial \theta^{\alpha} \rightarrow \mathrm{i} a^{2} g^{-1} \mathscr{E}^{\alpha}{ }_{l}$, where $\mathscr{E}^{\alpha}{ }_{l}$ denotes the chromoelectric field, and the relations (5a) and ( $5 b$ ), one sees that, in the $a \rightarrow 0$ limit, $H_{\mathrm{G}}$ is the sum of the squares of the (chromo-)electric and magnetic fields. I.e., the theory associated to the SDE (1) has (pure gauge) quantum chromodynamics as its classical continuum limit.

A necessary condition for the existence of a continuum limit for the euclidean quantum theory is existence of a coupling constant value $g^{*}$ where the correlation length diverges and a scaling transformation $g=g(a)$ such that physical quantities remain constant when $a \rightarrow 0$ and $\lim _{a \rightarrow 0} g(a)=g^{*}$.

To obtain an equation with diffusion coefficient independent of the lattice spacing one makes a scale transformation in the time variable $s \rightarrow \tau=s / a$ :

\#2 This is also called the Schrödinger representation of the theory [6]. Here one uses the name Dirichlet representation for the space with the ground state measure, Schrödinger representation being reserved for the space with flat measure obtained from $\mathscr{H}_{\mathrm{D}}$ by a unitary transformation. 
$\mathrm{d} \theta^{\alpha},(a \tau)=\mathrm{i} a b^{\alpha}{ }_{l} \mathrm{~d} \tau+g \mathrm{~d} W^{\alpha}{ }_{l}(\tau)$.

The correlation length is the inverse of the mass gap (lowest positive eigenvalue). If the critical point we are looking for is at $g^{*}=0$, then one may use the fact that the lowest positive eigenvalue for the boundary value problem of an elliptic operator $\left(H_{\mathrm{D}} u=\lambda u ;\left.u\right|_{\partial \mathrm{D}}=0\right)$ may be estimated at weak coupling (weak noise) from the theory of small random perturbations of dynamical systems $[7,8]$.

To obtain the smallest eigenvalue one chooses the domain $\mathrm{D}$ for the eigenvalue problem in such a way that $F\left(\sigma_{\mathrm{G}}\right)$ reaches its maximum and minimum values in $\mathrm{D}$. From the properties of the solutions of eq. (12) and the results of ref. [7] or ref. [8], applied to the case where the drift is the gradient of a function, one obtain for small $g$ (cf. theorem 11.1 of ref. [8] or theorem 7.4 of ref. [7])

$a m_{\mathrm{g}} \sim \exp \left[-2\left(F_{\max }-F_{\min }\right) / g^{2}\right]$.

For the physical mass gap to remain fixed when $a \rightarrow 0$ one requires

$g^{2}(a) \sim\left|2\left(F_{\max }-F_{\min }\right) / \log a\right|$.

Therefore when $a \rightarrow 0$ one obtains $g(a) \rightarrow 0$, consistent with the use of the small random perturbation results to compute (13). This establishes the existence of a scaling limit for the mass gap. We have therefore proved the following theorem.

\section{Theorem}

The SDE (1) with the drift given by eqs. (3) and (4) and the boundedness conditions on $F\left(\sigma_{\mathrm{G}}\right)$ defines a lattice euclidean theory for (chromodynamic) non-abelian gauge fields with a scaling limit for the mass gap $g(a) \rightarrow 0$ at $a \rightarrow 0$.

\section{Remarks}

(1) Had we chosen $F\left(\sigma_{\mathrm{G}}\right)=\sigma_{\mathrm{G}}$, i.e. $F\left(\sigma_{\mathrm{G}}\right)$ not bounded to values independent of the volume $V$ of the lattice $\Lambda_{a}$, we would have obtained

$a m_{\mathrm{g}} \sim \exp \left(-c^{\prime} V / g^{2} a^{3}\right)$

because $\sigma_{\mathrm{G}}$ grows with the number of links in the domain $A_{a}$. Then, to keep $m_{\mathrm{g}}$ fixed

$g^{2}(a) \sim\left|1 / a^{3} \log a\right|$

and $g(a) \rightarrow \infty$ when $a \rightarrow 0$, which is inconsistent with the $g \rightarrow 0$ hypothesis used to compute (15a).

(2) Using the volume-independent (upper and lower) boundedness of $F\left(\sigma_{\mathrm{G}}\right)$ one obtains a consistent scaling limit, no matter whether the gauge group is abelian or non-abelian. The choice of the model appropriate to each physical situation requires therefore some other considerations.

As explained in ref. [3], because $\sigma_{\mathrm{G}}$ is proportional to the Pontryagin index it seems physically reasonable to impose periodicity on $F\left(\sigma_{\mathrm{G}}\right)$, choosing the period in such a way that all the homotopically distinct Yang-Mills vacua have a similar drift in the SDE (1). On the other hand for U(1) there is only one class of vacuum configurations. Hence, one has no physical reason not to choose $F\left(\sigma_{\mathrm{G}}\right)=\sigma_{\mathrm{G}}$ in this case.

With these choices one would obtain a scaling model at $g \rightarrow 0$ for QCD and a model without a scaling limit at $g \rightarrow 0$ for lattice QED. Notice, however, that, in the latter case, when $V \rightarrow \infty$ at fixed $a$, eq. (15a) implies $m_{\mathrm{g}} \rightarrow 0$; i.e., the infinite volume lattice theory has no mass gap. Left open is therefore the possibility of existence of a scaling limit at some other $g \neq 0$.

The fact that, to obtain a well-defined theory, one needs to make these choices in the model, is a good illustration of the question raised in the introduction concerning the basic ambiguity of hamiltonian (or lagrangian) specifications of gauge theories. 
Now, one includes fermion fields in the stochastic description. A general scheme to write stochastic equations of fermions and gauge fields was developed in ref. [2]. The main conclusion is that euclidean evolution is, in this case, described by a stochastic process involving diffusion in the gauge coordinates and jumping between the fermion configurations.

Here one obtains an explicit form for the drift and transition functions in such a way that the generator of the process coincides, in the $a \rightarrow 0$ limit, with the QCD hamiltonian.

The fermion lattice fields $\psi(n)$ and $\bar{\psi}(n)$ are treated as coordinates in the same footing as $\theta^{\alpha}{ }_{1}$. It is only in the last step, after drift and transition functions are obtained, that $\psi$ and $\bar{\psi}$ are evaluated, by changing to an occupation number basis.

For Wilson fermions the standard lattice hamiltonian is

$$
\begin{aligned}
H_{\mathbf{F}} & =-\frac{\mathrm{i}}{2 a} \sum_{n, i} \bar{\psi}(n) \gamma^{i}\left[U_{n, n+i} \psi(n+i)-U_{n, n-i} \psi(n-i)\right] \\
& +\frac{B}{2 a} \sum_{n, i} \bar{\psi}(n)\left[2 \psi(n)-U_{n . n+i} \psi(n+i)-U_{n . n-i} \psi(n-i)\right]+m \sum_{n} \bar{\psi}(n) \psi(n) .
\end{aligned}
$$

\section{Define}

$$
\begin{aligned}
\sigma_{\mathrm{F}} & =-\frac{\mathrm{i}}{2 a} \sum_{n, i} \bar{\psi}(n) \gamma^{i}\left[\chi\left(\theta_{n, n+i}\right) \psi(n+i)-\chi\left(\theta_{n, n-i}\right) \psi(n-i)\right] \\
& -\frac{B}{2 a} \sum_{n, i} \bar{\psi}(n)\left[\chi\left(\theta_{n, n+i}\right) \psi(n+i)+\chi\left(\theta_{n, n-i}\right) \psi(n-i)\right]+\frac{1}{3}\left(m+\frac{B}{a}\right) \sum_{n, i} \bar{\psi}(n) \psi(n) \zeta\left(\theta_{n, n+i}\right),
\end{aligned}
$$

where

$\chi\left(\theta_{n, n+i}\right)=\frac{2 a}{g^{2}} \sum_{\alpha}\left[\left(\frac{1}{2} \theta_{n, n+i}^{\alpha}\right)^{2}+\frac{1}{6} \mathrm{i}\left(\theta_{n, n+i}^{\alpha}\right)^{3}\right] \xi^{\alpha}$,

$\zeta\left(\theta_{n, n+i}\right)=\frac{a}{C_{N} g^{2}} \sum_{\alpha} \theta_{n, n+i}^{\alpha} \theta_{n, n+i}^{\alpha}$

with $C_{N}=1$ for $\mathrm{U}(1)$ and $C_{N}=N^{2}-1$ for $\operatorname{SU}(N)$.

It is easy to see that an hamiltonian of the form

$H=-\frac{g^{2}}{2 a} \sum_{l, \alpha}\left(\frac{\partial}{\partial \theta^{\alpha},}+\frac{\partial \sigma}{\partial \theta^{\alpha}{ }_{l}}\right)\left(\frac{\partial}{\partial \theta^{\alpha}{ }_{l}}-\frac{\partial \sigma}{\partial \theta^{\alpha}{ }_{l}}\right)$

with $\sigma=\sigma_{\mathrm{G}}+\sigma_{\mathrm{F}}$ has the same $a \rightarrow 0$ limit as $H_{\mathrm{G}}+H_{\mathrm{F}}\left[H_{\mathrm{G}}\right.$ being the pure gauge hamiltonian eq. (11)]. In eq. (20) the terms that do not vanish in the $\alpha \rightarrow 0$ limit come from $\sum\left(\partial \sigma_{\mathrm{G}} / \partial \theta^{\alpha}{ }_{l}\right)\left(\partial \alpha_{\mathrm{G}} / \partial \theta^{\alpha}{ }_{l}\right)$ for $H_{\mathrm{G}}$ and from $\sum \partial^{2} \sigma_{\mathrm{F}} / \partial \theta^{\alpha},^{2}$ for $H_{\mathrm{F}}$.

The stochastic process is now constructed in such a way that its generator is

$$
\exp \left\{-\left[g^{2}\left(\sigma_{\mathrm{G}}+\sigma_{\mathrm{F}}\right) / a\right]\right\} H\left\{\exp \left[g^{2}\left(\sigma_{\mathrm{G}}+\sigma_{\mathrm{F}}\right) / a\right]\right\} \text {. }
$$

For the time evolution of the probability density of the process one obtains

$$
\begin{aligned}
& \frac{\partial}{\partial t} \rho(\theta, \psi, \bar{\psi})=\frac{g^{2}}{2 a} \sum_{\alpha, l} \frac{\partial^{2}}{\partial \theta^{\alpha}{ }_{l}^{2}} \rho-\sum_{\alpha, l} \frac{\partial}{\partial \theta^{\alpha}{ }_{l}}\left(\mathrm{i} b^{\alpha}, \rho\right) \\
& \quad+\sum_{\psi^{\prime} \bar{\psi}^{\prime}}\left[J\left(\theta \psi \bar{\psi} \mid \theta \psi^{\prime} \bar{\psi}^{\prime}\right) \rho\left(\theta, \psi^{\prime}, \bar{\psi}^{\prime}\right)-J\left(\theta \psi^{\prime} \bar{\psi}^{\prime} \mid \theta \psi \bar{\psi}\right) \rho(\theta, \psi, \bar{\psi})\right],
\end{aligned}
$$


$\rho=\rho(\theta, \psi, \bar{\psi})$ being the probability of the configuration $(\theta, \psi, \bar{\psi}) ;$

$\mathrm{i} b^{\alpha}{ }_{l}=\left(g^{2} / a\right)\left(\partial / \partial \theta^{\alpha}\right) \sigma$

and $J$ is the jumping transition function, to be defined below.

So far one has considered $\psi(n)$ and $\bar{\psi}(n)$ as classical coordinates in the same footing as $\theta^{\alpha}{ }_{1}$ In the present form eq. (21) is only of formal interest. For computation purposes one has to evaluate the fermion coordinates in each configuration.

The generator (hamiltonian) of the process being time invariant one may use a $(t=0)$ free field expansion of $\psi$ and $\bar{\psi}$ and evaluate arbitrary functions of the fermion coordinates by its expectation values in an occupation number basis

$F(\theta \psi \bar{\psi}) \rightarrow F(\theta f \bar{f})=\langle f \bar{f}|F(\theta \psi \bar{\psi})| f \bar{f}\rangle$.

For details on the occupation number basis to be used see ref. [2].

Note that because the occupation number basis is constructed from operators defined at $t=0$, the configurations $(f \bar{f})$ are not configurations of real physical fermions, although the basis $|\theta f \bar{f}\rangle$ is a quite legitimate one to describe the evolution of the process.

With the replacement (23) the stochastic eq. (21) becomes

$\frac{\partial}{\partial t} \rho(\theta f \bar{f})=\frac{g^{2}}{2 a} \sum_{\alpha, l} \frac{\partial^{2}}{\partial \theta^{\alpha}{ }_{l}^{2}} \rho(\theta f \bar{f})-\sum_{\alpha, l} \frac{\partial}{\partial \theta_{l}^{\alpha}{ }_{l}}\left(\mathrm{i} b^{\alpha}, \rho\right)+\sum_{f^{\prime}, f^{\prime}}\left[J\left(\theta f \bar{f} \mid \theta f^{\prime} \bar{f}^{\prime}\right) \rho\left(\theta f^{\prime} \bar{f}^{\prime}\right)-J\left(\theta f^{\prime} \bar{f}^{\prime} \mid \theta f \bar{f}\right) \rho(\theta f \bar{f})\right]$

with $\sigma$, in the drift definition, given by

$\sigma=\sigma_{\mathrm{G}}+\left\langle f \bar{f}\left|\sigma_{\mathrm{F}}\right| f \bar{f}\right\rangle$

and

$J\left(\theta f \bar{f} \mid \theta f^{\prime} \bar{f}^{\prime}\right)=-\phi^{-1}\left(\theta f^{\prime} \bar{f}^{\prime}\right)\left\langle\theta f^{\prime} \bar{f}^{\prime}\left|V_{\mathbf{R}}\right| \theta f \bar{f}\right\rangle \phi(\theta f \bar{f})$,

$\phi(\theta f \bar{f})=\exp \left(g^{2} \sigma_{\mathrm{G}} / a\right)\left\langle\theta f \bar{f}\left|\exp \left(g^{2} \sigma_{\mathrm{F}} / a\right)\right| \theta f \bar{f}\right\rangle$,

$V_{\mathrm{R}}=\frac{g^{2}}{2 a} \sum_{\alpha, l} \frac{\partial^{2}}{\partial \theta^{\alpha}{ }_{l}^{2}} \sigma_{\mathrm{F}}+\frac{g^{2}}{2 a} \sum_{\alpha, l} \frac{\partial \sigma}{\partial \theta^{\alpha}} \frac{\partial \sigma}{\partial \theta^{\alpha}}$.

Eq. (24) involves diffusion in the $\theta$ variables and jumping between fermion configurations. Fermion-boson coupling takes place through the dependence of the drift on the $(f \bar{f})$ configurations and the dependence of the jumping transition functions on $\theta$. Eq. (24) describes, in the same sense as eq. (1), and euclidean evolution for QCD-like fields. A proof of existence of a pathwise unique solution defining a Dirichlet representation, Schwinger functions and mass gap scaling (as in the pure gauge case) seems within reach and is in progress.

In conclusion, stochastic differential equations seem to be a promising tool to construct non-trivial quantum field theories in spacetime dimension four. In this connection one should also mention the important work of Albeverio and Hoegh-Krohn [9] and Haba [10].

\section{References}

[ 1 ] S.M. Eleutério and R. Vilela Mendes, Stochastic mechanics of abelian lattice theories, J. Phys. A 20 (1987), to be published.

[2] S.M. Eleutério and R. Vilela Mendes, Z. Phys. C 34 (1987) 451.

[3] R. Vilela Mendes, Non-equivalent stochastic models in lattice gauge theory, preprint IFM-3/87, Phys. Rev. D, to be published.

[4] N. Ikeda and S. Watanabe, Stochastic differential equations and diffusion processes (North-Holland, Amsterdam, 1981 ).

[5] R.Z. Khas'minskii, Stochastic stability of differential equations (Sijthoff \& Noordhoff, Alphen aan den Rijn, 1980). 
[6] J. Glimm and A. Jaffe, Quantum physics, 2nd Ed. (Springer, Berlin, 1987) ch. 6.

[7] M.I. Freidlin and A.D. Wentzell, Random perturbations of dynamical systems (Springer, Berlin, 1984) ch. 6,

[8] A. Friedman, Stöchastic differential equations and applications, Vol. 2 (Academic Press, New York, 1976), ch. 14.

[9] S. Albeverio and R. Høegh-Krohn, Phys. Lett. B 177 (1986) 175; B 189 (1987) 329.

[10] Z. Haba, in: Stochastic processes in classical and quantum physics, eds. S. Albeverio, G. Casati and D. Merlini, Lecture Notes in Physics, Vol. 262 (Springer, Berlin, 1986). 